pement de la cavitation. Les conséquences de ce choix doivent être évaluées avec précision. La fourniture d'énergie de pointe peut s'accommoder de turbines ayant une durée de vie (sans réparations) moindre, si l'investissement qu'elles représentent est réduit du fait de l'acceptation d'un certain développement de la cavitation. Le dimensionnement d'une hélice de super-tanker fait appel à des notions similaires. La présence de cavitation développée lors d'un transitoire peut être tolérée si elle conduit à l'amélioration du fonctionnement en régime nominal: décolage, puis croisièrc d'un hydroptère, par exemple.

La complexité des écoulements nécessite le plus souvent encore l'emploi de l'expérimentation pour connaître les champs de vitesse et de pression; a fortiori, l'étude de la cavitation doit-elle faire appel à l'expérience physique. On veut non seulement connaître la limite d'apparition de la cavitation, mais aussi prévoir le fonctionnement, au-delà de cette limite, en cavitation développée.

Pour qualifier les méthodes expérimentales, on a procédé à des échanges entre laboratoires de modèles de turbines, on a testé le même tracé d'ogive dans de nombreux tunnels à hélice marine; la dispersion des résultats obtenus reste, en dépit du soin apporté aux essais, incompatible avec les exigences actuelles des applications industrielles. Une analyse des causes de cette dispersion met en évidence l'im- portance des propriétés des fluides utilisés, la propension d'un fluide à caviter étant difficilement reproductible d'un essai à l'autre.

Les zones de rupture préférentielle de la continuité du fluide sont constituées par de micro-domaines gazeux appelés germes ou noyaux de cavitation; leur nombre et leur taille conditionnent la nucléation du fluide et, dans une certaine mesure, l'expansion des cavités gazeuses élémentaires.

L'analyse du phénomène intime et la formulation des lois élémeritaires nécessitera sûrement encore un effort soutenu; pour répondre au besoin pressant de qualification de la propension à caviter d'un fluide, de nombreux auteurs ont pensé à une approche globale qui devrait permettre de qualifier un fluide par un paramètre simple; cette démarche scientifique peut donner dans certains cas d'excellents résultats; on a pu industriellement définir et mesurer une température bien avant d'avoir fait l'analyse thermodynamique de sa signification profonde.

La notion de pression critique fait appel à ceite démarche globale; son attrait est évident et a séduit de nombreux auteurs; malheureusement on montrera qu'il n'est pas possible de qualifier un fluide par la pression critique telle que nous allons la définir.

\section{Notion de pression critique de cavitation développée}

On veut caractériser l'ensemble des propriétés d'un fluide, en ce qui concerne la cavitation développée, par un paramètre unique, lui-même fonction de l'ensemble des paramètres élémentaires caractéristiques du fluide.

Le paramètre représentatif des propriétés du fluide peut avoir plusieurs dimensions et être introduit dans la similitude de différentes manières.

Lorsque l'on considère la dynamique de la formation de la cavitation, on constate l'importance des microbulles de gaz libre; de leur stabilité dépend l'apparition et le développement de la cavitation. On sait (Rayleigh) que ces bulles deviennent instables pour une dépression caractéristique de la masse de gaz qu'elles contiennent. De même, si l'on considère le paramètre de Thoma, c'est une différence de pression qui caractérise le développement de la cavitation.
Ces considérations incitent à choisir comme paramètre caractéristique du fluide un paramètre scalaire à distribution uniforme ayant les dimensions d'une pression.

Cependant, il ne faut confondre ce paramètre « pression critique » ni avec la pression d'instabilité des bulles at sens de Rayleigh, ni avec une quelconque pression locale prise dans l'écoulement. Il s'agit bien d'un paramètre voulant caractériser le fluide, et rien que le fluide, en faisant abstraction d'une configuration particulière d'écoulement.

On suppose aussi qu'en remplaçant la pressios de vapeur dans le paramètre de Thoma par la pression critique, on disposerait d'un paramètre de transposition permettant de prendre en compte un changement éventuel du fluide.

\section{Approche expérimentale}

\section{Choix des essais.}

On a voulu voir s'il était possible de trouver une corrélation entre une éventuelle pression critique et les « performances $\gg$ de différentes configurations. Les configurations d'écoulement choisies schématisent les types d'écoulement rencontrés fréquemment dans les applications.

On a retenu deux configurations bidimensionnelles planes (une aile, un profil en paroi), une configuration bidimensionnelle de révolution (disque en conduite).

Les efforts hydrodynamiques sur l'aile fournissent un critère quantifiable du développement de la cavitation. Des mesures de pression locale dans la zone affectée par la cavitation étaient effectuées dans toutes les configurations.

\section{Moyens d'essais.}

Les veines contenant les modèles ont été incorporées dans le courant d'un tunnel de cavitation pressurisable ayant un débit potentiel de $0,3 \mathrm{~m}^{3} / \mathrm{s}$.

Sans décrire en détail le tunnel employé, on souligne l'importance des deux larges cuves à surface libre qui limitent à un faible niveau le bruit dans la veine d'essai.

On remarque aussi l'organe à perte de charge ajustable et non cavitant qui permet, pour un même sigma de Thoma dans la veine d'essai, de faire varier la teneur en gaz dissous par le réglage de la pression dans la cuve aval.

A part les mesures des caractéristiques aux limites des modèles, on a utilisé des capteurs en paroi situés dans les zones affectées par la cavitation; l'aile était montée sur une balance à trois composantes. 


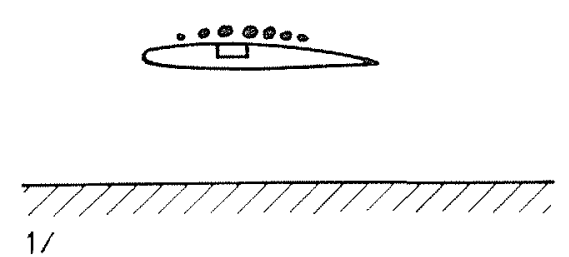

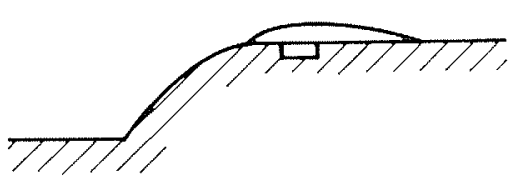

2/

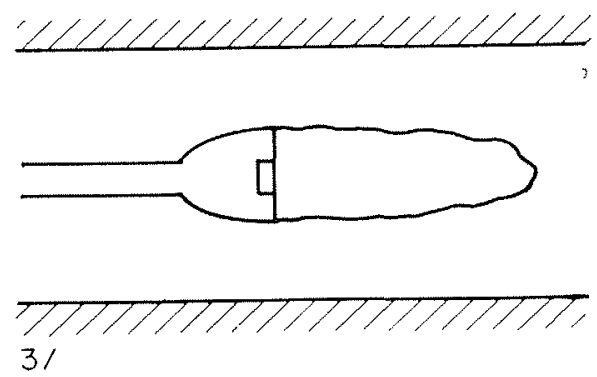

$1 /$ Configurations Étudí́is. - 1/ Aile bidimensionnelle; $2 /$ Profil en paroi; 3/ Forme de révolution.

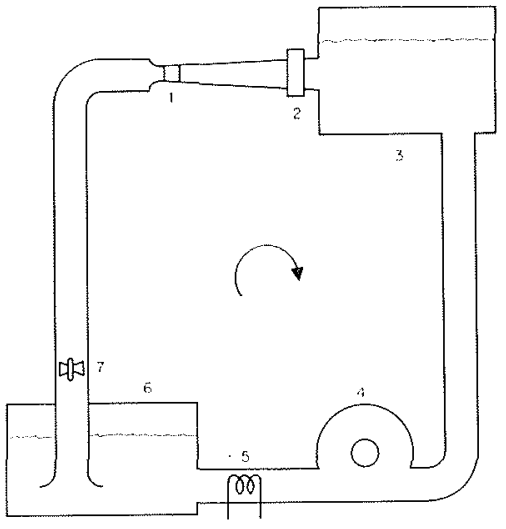

2/ Schéma du tunnel. - 1/ Veine d'essai; 2/ Organe de perte de charge; $3 /$ Cuve aval; 4/Pompe; 5/ Echangeur; 6/ Cl1ve amont; 7/ Débitmètre.

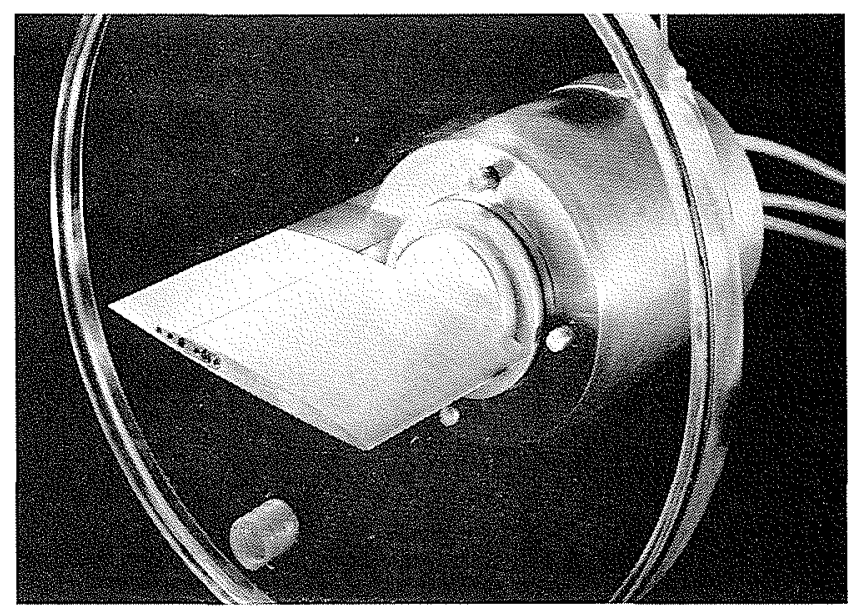

Photo 2/ Aile bidimensionnelle en balance.

\section{Résultats principaux.}

On a constaté :

- dans les zones affectées par la cavitation, la pression locale était toujours égale à la pression de vapeur. (à $\pm 2 \mathrm{~cm}$ d'eau près);

- que, pour un même sigma de Thoma, la portance d'une aile pouvait varier de manière importante suivant l'origine de l'eau (cycle de dégazage différent, mais de même teneur en air dissous).

Par exemple, pour sigma correspondant à une perte de portance de $50 \%$, avec une eau $A$, on n'avait que $35 \%$ de perte avec une eau B. Cet exemple extrême laisse entrevoir l'importance de la nature intime du fluide.

Interprétation des résultats

En prenant :
$P$ : pression de référence;
$P_{M}$ : pression locale en $M$;
$V$ : vitesse de référence;
$p:$ masse spécifique;
$\mathrm{P}_{v}:$ pression de vapeur;
$\mathrm{P}_{c r}$ : pression critique.

Pour un point $\mathrm{M}$ donné dans un écoulement:

$$
\left(\mathrm{P}-\mathrm{P}_{\mathrm{M}}\right) / 1 / 2 \rho \mathrm{V}^{2} \text { est un invariant. }
$$

Si le point $M$ est dans la zone affectée par la cavitation, $\mathrm{P}_{\mathrm{M}}=\mathrm{P}_{v}$, la quantité $\left(\mathrm{P}-\mathrm{P}_{v}\right) / 1 / 2 \mathrm{pV}^{2}$ est donc un invariant.

De même, la notion de pression critique entraîne:

$$
\left(\mathrm{P}-\mathrm{P}_{c r}\right) \mathrm{p} / \mathrm{\rho} \mathrm{V}=\text { invariant }
$$

3/ A/ Relation entre la teneur en oxygène dissous et la pression dans la cuve aval.

B/ Deux historiques possibles de teneur en oxygène dissous aboutissant à la même valeur finale.

C/ Evolution de la portance d'un profil en fonction du sigma de Thoma nour les caux obtenues suivant les historiques en $B$.
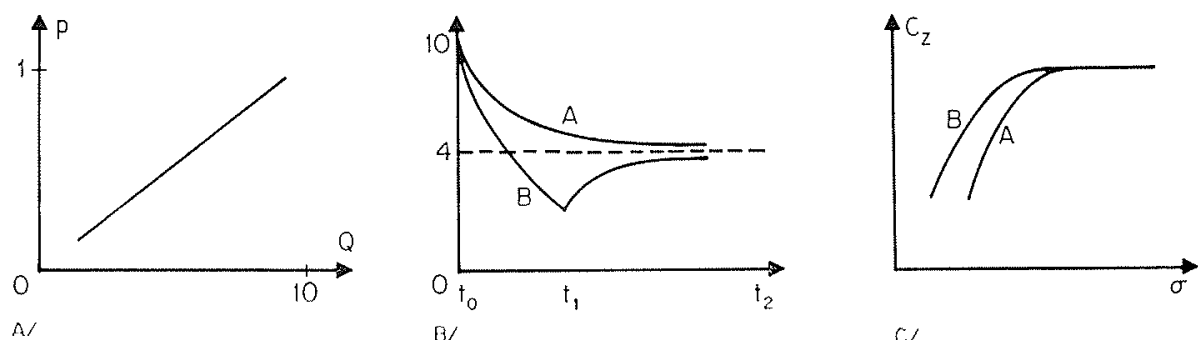

B/ 


\section{F DANEL}

Les invariants $\left(\mathrm{P}-\mathrm{P}_{v}\right) / 1 / 2 \mathrm{\rho} \mathrm{V}^{2}$ et $\left(\mathrm{P}-\mathrm{P}_{(r)}\right) / 1 / 2 \mathrm{pV} \mathrm{V}^{2}$ ne sont compatibles que si $\mathrm{P}_{c r}=\mathrm{P}_{v}$.

Or, pour un même sigma de Thoma $\left(\mathrm{P}-\mathrm{P}_{1}\right) / 1 / 2 \mathrm{PV}^{*}$, on constate qu'une aile peut avoir des portances différentes suivant l'origine de l'eau utilisée; la pression critique ne peut donc pas caractériser l'eau utilisée.

On constate aussi que les variations de portance $\Delta \mathrm{F}$ et de moment $\Delta \mathrm{M}$ que l'on obtient en changeant l'origine de l'eau utilisée, sont liées par une relation:

$$
\Delta \mathrm{M}=\mathrm{L} \Delta \mathrm{F}
$$

où $\mathrm{L}$ est une constante qui représente la distance du barycentre des variations de pression sur le profil à l'axe de référence de la balance.

\section{Remerciements}

L'auteur remercie tout particulièrement $M$. J. Duport pour ses conseils attentifs, M. J. P. Bertrand pour l'accueil réservé au sein d'une équipe qui a collaboré efficacement à ces études.

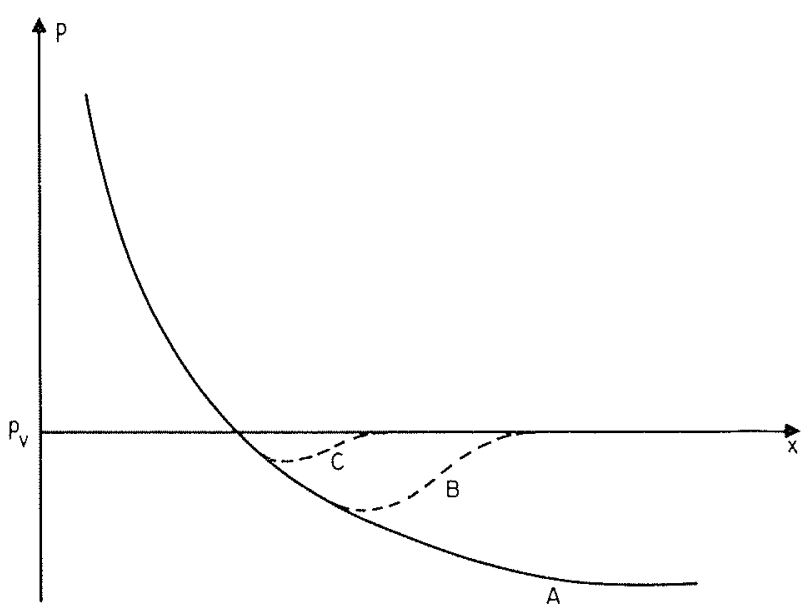

4/ Evolution possible de la pression en fonction de labscisse dans la zone dinitiation de la cavitation, suivant la taille des germes de cavitation: A/ sans germes; $\mathrm{B} /$ petits germes; C/ gros germes. Ceci est une image qui demanderait à être précisée.
S. BINDEL et R. LAMBARdo. - Influence de la vitesse et de la teneur en air de l'eau sur l'apparition de la cavitation sur modèle, ATMA, 1964.

S. Bindel et J.C. Riou. - (Suite de l'étude précédente), ATMA, 1969.
H. Hingken et C. A. Johnsson. - Cavitation inception on head form ITTC comparative experiments, Annexe $V$ au rapport du Comité de cavitation à la $11^{\circ}$ ITTC, Tokyo, 1966.

J.P. DuporT, - La cavitation de mélange, S.H.M., 1967.

F. DANel et J.P. Duport. - Characterisation of water in terms of fully developed cavitation, Forum de cavitation, A.S.M.E., 1969. 